\title{
Energy-Loss Calculation of Gain in a Plane Sinusoidal Free-Electron Laser
}

\author{
ROBERTO COISSON
}

\begin{abstract}
The gain of a free-electron laser (FEL) made with a plane sinusoidal undulator is calculated by the electron beam energy loss.
\end{abstract}

$\mathrm{T}$ HE free-electron laser (FEL) consists of an undulator (periodic transverse magnetic field) where an EM wave coming in the same direction as the ultrarelativistic $\left(\gamma^{2} \gg 1\right)$ electrons stimulates the emission of radiation.

The gain of an FEL can be calculated classically by the energy lost by the electron beam in the combined field of the undulator and of the input wave [1]-[4]. All of these calculations have only considered the case of the helical undulator.

It has also been remarked that there is a general relationship between gain and spontaneous spectrum for a free-electron device, independent of the particular undulator structure and allowing the gain of an arbitrary FEL to be calculated without describing the dynamics of the electrons in the device. In this way, the gain for a plane sinusoidal undulator has also been obtained [6]-[9].

As most of the experiments now in progress (e.g., on ACO and ADONE [10]) use plane sinusoidal undulators, it is of interest to show that the same result can be obtained by an energy-loss calculation, which better illustrates the physical origin of the differences between the two cases, and shows the more complex dynamics of the electrons in this case, which is not described by a simple pendulum potential.

We are considering an undulator

$$
\vec{B}=\hat{y} B_{0} \sin \frac{2 \pi}{\lambda_{0}} x
$$

of length $L=N \lambda_{0}$, with an electron beam of current $I$, electron energy $\gamma m c^{2}$, and effective cross-sectional area $\sigma$ (including filling factor).

Let us first summarize the derivation of the gain from the gain-spectrum relationship

$$
G=\frac{2 \gamma^{2}}{m c^{2}} \frac{d}{d \gamma} \frac{d W(\theta=0)}{d \Omega d \gamma d \sigma}
$$

where $d W / d \Omega d \nu$ is the spontaneous power per unit solid angle and bandwidth (a function of $\nu=c / \lambda$ and depending on $\gamma$ ).

The spectrum of incoherent emission from a plane sinusoidal undulator at $\theta=0$ (forward direction) has been calculated by

Manuscript received December 7, 1980.

The author was with the Istituto di Fisica, Università di Parma, Parma, Italy. He is now with the SPS Division, CERN, 1211 Geneve 23 , Switzerland.
Alferov, Bashmakov, and Bessonov [11]: for the $n$th harmonic we have

$$
\begin{aligned}
\frac{d W(\theta=0)}{d \Omega d \nu}= & \frac{2 \pi e}{c} I \gamma^{2} N^{2} \frac{b^{2} n^{2}}{1+\frac{1}{2} b^{2}} \\
& \cdot\left[J_{(n-1) / 2}(n \epsilon)-J_{(n+1) / 2}(n \epsilon)\right]^{2}
\end{aligned}
$$

where $b=\left(e B_{0} \lambda_{0} / 2 \pi m c\right)=\alpha \gamma$ is the "deflection parameter" (MKS units, $\alpha$ maximum deflection, $\alpha^{2} \ll 1$ )

$$
\xi=\frac{\pi}{2} \frac{\delta \nu}{\Delta \nu},
$$

where $\delta \nu$ is the detuning from peak of spontaneous spectrum and $\Delta \nu$ is the spontaneous bandwidth $\sim 1 / N$,

$$
\epsilon=\frac{\frac{1}{4} b^{2}}{1+\frac{1}{2} b^{2}}
$$

and $J$ are Bessel functions.

As $(d / d \gamma)=(\pi N / \gamma)(d / d \xi)$, from (1) and (2) we find, immediately, the single-pass gain on the $n$th harmonic $(n=1,3,5$, $7 \cdots)$ :

$$
\begin{aligned}
G_{n}= & \pi^{2} \frac{I}{\sigma I_{A}} f(\xi) \frac{N^{3} \lambda_{0}^{2}}{\gamma^{3}} b^{2} M_{n}(b)=4 \pi^{2} \frac{I}{\sigma I_{A}} \\
& \cdot f(\xi) \gamma N^{3} \lambda^{2} \frac{b^{2} n^{2}}{\left(1+\frac{1}{2} b^{2}\right)^{2}} M_{n}(b) \\
= & G_{H}(b / \sqrt{2}) M_{n}(b)
\end{aligned}
$$

where

$$
M_{n}(b)=\left[J_{(n-1) / 2}(n \epsilon)-J_{(n+1) / 2}(n \epsilon)\right]^{2}
$$

and $G_{H}$ is the gain of a helical FEL, $I_{A}=m c^{3} / e \simeq 17 \mathrm{kA}$, and

$$
f(\xi)=\frac{d}{d \xi}\left(\frac{\sin \xi}{\xi}\right)^{2}
$$

which has a maximum value $\simeq 0.54$; more generally, $f(\xi)$ is the derivative of the modulus square of the Fourier transform of the undulator.

Coming now to the energy-loss calculation, we have to find the maximum relative energy gain per unit length $\delta \gamma_{s}^{\max } / \gamma_{s}$ for a "synchronous" electron (i.e., an electron which "sees" the undulator and the input wave $E_{i}$ as having the same frequency, or for which the input wave is tuned at the peak of the spontaneous spectrum). Considering a general monoenergetic beam and averaging the energy lost in the distance 
$L$ over the initial positions of the electrons, the gain per pass will be

$$
G=\frac{\gamma m c^{2}\langle\delta \gamma / \gamma\rangle}{(c / 4 \pi)\left\langle E_{i}^{2}\right\rangle \sigma} \frac{I}{e}=\frac{2 \pi^{2} m c}{e \sigma\left\langle E_{i}^{2}\right\rangle}\left(\frac{\delta \gamma_{s}^{\max }}{\gamma_{s}}\right)^{2} I N L^{2} \gamma f(\xi)
$$

as

$\left\langle\frac{\delta \gamma}{\gamma}\right\rangle=\frac{\pi}{2}\left(\frac{\delta \gamma_{s}^{\max }}{\gamma_{s}}\right)^{2} L^{2} N f(\xi), \quad$ with $\xi=\pi N \frac{\gamma-\gamma_{s}}{\gamma_{s}}=\frac{\pi}{2} \frac{\delta \gamma}{\Delta \gamma}$.

Let us find $\delta \gamma_{s}^{\max } / \gamma_{s}$ in our case [(1)] .

For an electron with velocity $v \simeq c\left(1-\frac{1}{2} \gamma^{2}\right)\left(\gamma^{2} \gg 1\right)$, with suitable initial conditions

$$
\begin{aligned}
\frac{d z}{d t} & =\frac{c b}{\gamma} \cos \omega_{0} t \quad\left(\omega_{0}=2 \pi c / \lambda_{0}\right) \\
F_{z}(t) & =e E_{i} \cos \omega\left(t-\frac{x(t)}{c}\right) \\
x(t) & =\left(1-\frac{1+\frac{1}{2} b^{2}}{2 \gamma^{2}}\right) c t-\frac{\lambda_{0}}{16} \frac{b^{2}}{\gamma^{2}} \sin 2 \omega_{0} t .
\end{aligned}
$$

Let

$$
\omega_{1}=\frac{\omega}{2 \gamma^{2}}\left(1-\frac{1}{2} b^{2}\right) .
$$

We remark that the longitudinal oscillation amplitude is comparable with the output wavelength $\lambda=2 \pi c / \omega$ :

$$
\begin{aligned}
\frac{\delta \gamma_{s}^{\max }}{\gamma_{s}}= & \frac{1}{\gamma m c^{3}}\left\langle F_{z} \frac{d z}{d t}\right\rangle=\frac{e E_{i} b}{\gamma^{2} m c^{2}} \\
& \cdot\left\langle\cos \omega_{0} t \cos \left(\omega_{1} t+\eta \sin 2 \omega_{0} t\right)\right\rangle
\end{aligned}
$$

where

$$
\eta=\frac{1}{8} \frac{\lambda_{0}}{\lambda} \frac{b^{2}}{\gamma^{2}}
$$

where \langle\rangle represents time average for a given electron. Remembering that

$$
\begin{aligned}
\cos \left(\omega_{1} t+\eta \sin 2 \omega_{0} t\right)=\cos \omega_{1} t \cos \left(\eta \sin 2 \omega_{0} t\right) \\
\quad-\sin \omega_{1} t \sin \left(\eta \sin 2 \omega_{0} t\right) \\
=\cos \omega_{1} t\left[J_{0}(\eta)+2 \sum_{1}^{\infty}{ }_{k} J_{2 k}(\eta) \cos 4 k \omega_{0} t\right] \\
\quad-\sin \omega_{1} t\left[2 \sum_{0}^{\infty}{ }_{j} J_{2 j+1}(\eta) \sin 2(2 j+1) \omega_{0} t\right]
\end{aligned}
$$

and that the average value of the products of trigonometric functions is $\frac{1}{4}\left(\frac{1}{2}\right.$ for the $J_{0}$ term) if $\omega_{i}=n \omega_{0}$, with $n=4 k \pm 1$ or $n=4 j+2 \pm 1$, that is, with $n$ odd positive integer, and $=0$ otherwise. We can write $\eta=n \epsilon$ with $\epsilon=\left[\frac{1}{4} b^{2} /\left(1+\frac{1}{2} b^{2}\right)\right]$. The single-pass gain $G_{n}$ on the $n$th harmonic $(n=1,3,5,7$, $\cdots$ ), substituting (10) and (11) into (6), is then identical to (4).

This is for an energy spread $\Delta \gamma \ll \gamma / 2 N$ and angular spread $\Delta \theta \ll\left[\left(1+\frac{1}{2} b^{2}\right) / \sqrt{N} \gamma\right]:$ for a beam with nonnegligible $\Delta \gamma$ and $\Delta \theta$, the result has to be convoluted with a longitudinal velocity spread distribution corresponding to the energy and angular distributions.

\section{ACKNOWLEDGMENT}

The author wishes to thank Y. Farge, G. Brautti, D.A.G. Deacon, and E. G. Bessonov for discussions and useful information.

\section{REFERENCES}

[1] R. B. Palmer, "Interaction of relativistic particles and free e.m. waves in the presence of a static helical magnet," J. Appl. Phys., vol. 43, pp. 3014-3023, 1972.

[2] W. B. Colson, "Theory of a FEL," Phys. Lett., vol. 64A, p. 90, 1977.

[3] D. F. Alferov and E. G. Bessonov, "Classical theory of induced e.m. radiation from charged particles in an undulator," Sov. Phys. Tech Phys., vol. 24, pp. 450-455, 1979; D. F. Alferov, Yu. A. Bashmakov, and E. G. Bessonov, Preprint 163, FIAN, Moscow, 1976.

[4] A. Bambini and A. Renieri, "The FEL: A single-particle classical model," Lett. Nuovo Cim., vol, 21, pp. 399-404, 1978; A. Bambini, A Renieri, and S. Stenholm, "Classical theory of a FEL in a moving frame," Phys. Rev. A, vol. 19, pp. 2013-2022, 1979; C. Pellegrini, "The FEL: An introduction," in The Possible Impact of FELs on Spectroscopy and Chemistry, G. Scoles, Ed. Workshop Report, Riva del Garda, 1979; C. Pellegrini, "Physics of the FEL," to be published.

[5] F. A. Hopf, P. Meystre, M. O. Scully, and W. H. Louisell, "Classical theory of a FEL," Opt. Commun., vol. 18, pp. 413 416, 1976; - - "Strong signal theory of a FEL," Phys. Rev. Lett., vol. 37, pp. 1342-1345, 1976; V. N. Baier and A. I. Milstein, "To the theory of a FEL," Phys. Lett., vol. 65A, pp. 319-322, 1978 ; M. V. Fedorov and J. K. McIver, "Multiphoton stimulated Compton scattering," Opt. Commun., vol. 32, pp. 179-182, 1980 .

[6] A. A. Kolomenski and A. N. Lebedev, "Emission of undulator radiation from relativistic electrons and physical processes of 'electron lasers'," Kvantovaya Elektronika, vol. 5, pp. 1543$1551,1978$.

[7] J.M.J. Madey, "Relationship between mean radiated energy, mean squared radiated energy and spontaneous power spectrum in a power series expansion of the equation of motion in a FEL," Nuovo Cimento, vol. 50B, pp. 64-88, 1979.

[8] J.M.J. Madey and R. Taber, Physics of Quantum Electronics, vol. 8, S. F. Jacobs et al., Eds. Reading, MA: Addison-Wesley, 1980.

[9] D.A.G. Deacon, to be published.

[10] R. Barbini and G. Vignola, "LELA: A FEL experiment in Adone," Rep. LNF-80/12 (R), Frascati, 1980.

[11] D. F. Alferov, Yu. A. Bashmakov, and E. G. Bessonov, "Undulator radiation," Sov. Phys. Tech. Phys., vol. 18, pp. 1336-1339, 1974.

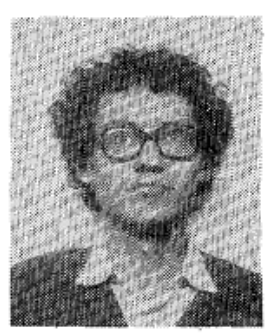

Roberto Coisson was born in Torre Pellice. Italy, in 1943. He received the "laurea in fisica" from the University of Florence, Florence, Italy, in 1966. His thesis was on laser technology.

He was in the Republic of Guinea for two years, teaching laboratory physics at the Institut Polytechnique de Conakry and working on construction of apparatus with local resources. He has taught electromagnetic waves and quantum electronics at the University of Lecce, Parma, Italy. He is presently a Scientific Associate at CERN, Geneva, Switzerland. His research interests have included physics education, construction of lasers, high voltage technology, projects of "undulators" for production of X-rays, and synchrotron radiation in nonuniform fields; in particular he proposed the possibility of observing visible synchrotron radiation from protons (dúe to "edge effect"), and participated, with a team of CERN Super Proton Synchrotron, to the first observation of this effect, which is now used to monitor high-energy proton beam profiles. 